\title{
O ctreótido, tratamiento endoscópico o ambos en la hemorragia variceal activa en pacientes cirróticos: estudio multicéntrico
}

\author{
G uillermo Silva $P^{1}$, Rodrigo $Q$ uera $P^{2}$, Fernando Fluxá ${ }^{1}$, \\ Edgar Sanhueza B ${ }^{3}$, Roberto Segovia M ${ }^{1}$, Javier Brahm B ${ }^{1,2}$, \\ Cristián Muñoz $\mathrm{T}^{3}$, Antonio Morales $\mathrm{B}^{2,3}$, Lorena Muñoz $\mathrm{C}^{4}$, \\ Luis U rzúa $\mathrm{F}^{4}$, Leandro Biagini $A^{5}$. \\ Octreotide administration and/or endoscopic \\ treatment in cirrhotic patients with acute \\ variceal bleeding: a multicentric study
}

Background: Acute variceal bleeding in cirrhotic patients is an emergency with a high risk of rebleeding and death. Endoscopic procedures such as sclerotherapy or banding, combined or not with drugs such as octreotide could be considered. Aim: To assess the value of octreotide in the control of acute variceal bleeding. Patients and methods: Ninetytwo patients were randomized into three groups: endoscopic therapy plus octreotide $50 \mu \mathrm{g} / \mathrm{h}$ bolus and continuous infusion for 5 days $(n=36)$; octreotide (same dosage) $(n=13)$ and endoscopic therapy only (banding and/or sclerotherapy) $(n=43)$. Results: Haemostasis at 24 hours was achieved in $97 \%$ of patients with combined treatment, $69 \%$ of patients receiving octreotide, and $93 \%$ of patients with endoscopic therapy $(p=0.2)$. Three patients with combined treatment, four patients receiving octreotide and eight patients with endoscopic therapy, rebled during the first five days $(p=0.15)$. The mean of blood units transfused was similar in the three groups. No differences were observed in hospital days and side effects. At 42 days of follow up, eight patients with endoscopic therapy, one patients with combined therapy and 2 patients receiving octreotide, died ( $p=N S$ ). Conclusions: Octreotide is useful in the management of acute variceal bleeding. The absence of important side effects, renders it as a safe adjuvant treatment associated with endoscopic treatment (Rev Méd Chile 2004; 132: 285-94).

(Key Words: Esophageal and gastric varices; Gastrointestinal hemorrhage; Hypertension, Octreotide; Portal)

Recibido el 3 de junio, 2003. Aceptado en versión corregida el 16 de diciembre, 2003.

${ }_{1}^{1}$ Departamento de Gastroenterología, Clínica Las Condes. ${ }^{2}$ Sección de Gastroenterología, Hospital Clínico de la Universidad de Chile. ${ }^{3}$ Servicio de Gastroenterología, Hospital Militar. ${ }^{4}$ Servicio de Farmacia y ${ }^{5}$ Centro de Epidemiología, Hospital Clínico de la Universidad de Chile. Santiago, Chile.

Correspondencia a: Dr. Guillermo Silva P. Departamento de Gastroenterología, Clínica Las Condes. Lo Fontecilla - Las Condes. Teléfono: 2104290. E-mail: GuillermoSilvap@entelchile.net 
L a hemorragia por várices esofágicas (HV) es una importante complicación de la hipertensión portal y su mortalidad oscila entre 25 y $50 \% 1-6$. El riesgo de resangrar dentro de las primeras 6 semanas es de aproximadamente $50 \%$, la mitad de estos casos ocurre dentro de las primeras $48 h^{1,2,7}$. Todo ello justifica que el manejo de la hemorragia consista no sólo en el control inmediato del sangrado, sino que también en prevenir la recurrencia, minimizar el deterioro de la función hepática y reducir las complicaciones asociadas con ella.

En el tratamiento de la HV se han utilizado terapias endoscópicas (escleroterapia o ligaduras), taponamiento con balón, farmacológicas, shunt porto-sistémico intrahepático transyugular (TIPS) y cirugías derivativas ${ }^{1-5,8-11}$. El más utilizado ha sido el endoscópico, con control del sangrado inmediato cercano a $90 \% 12,13$. En nuestro medio, Vargas y col han señalado que la ligadura elástica complementada con esclerosis en el tratamiento de urgencia, logra controlar el sangrado en más de $90 \%$ de los casos ${ }^{11}$.

Otros autores han señalado que el tratamiento farmacológico de la $\mathrm{HV}$ activa permitiría una mayor estabilización hemodinámica ${ }^{1-5}$ y que su asociación con escleroterapia disminuiría los episodios de resangrado precoz, requerimientos transfusionales y tiempo de hospitalización ${ }^{14,15}$. Uno de estos fármacos es el octreótido, análogo sintético de la somatostatina y cuyo principal mecanismo de acción en la HV sería la vasoconstricción esplácnica, al inhibir la liberación de hormonas vasodilatadoras como glucagón y sustancia $\mathrm{P}^{1-4}$. Sin embargo, existen datos contradictorios sobre su efecto en la presión portal y el flujo de la vena ácigos ${ }^{16,17}$. Además el octreótido reduce la hiperemia postprandial y aumentaría la agregación plaquetaria ${ }^{18-20}$. A su vez, sus efectos adversos son menores comparados con el uso de otros fármacos como vasopresina o terlipresina ${ }^{9,21}$.

En nuestro medio se desconoce la utilidad del octreótido como modalidad terapéutica, por lo que nuestros objetivos fueron compararlo con la terapia endoscópica (escleroterapia o ligaduras) sola o asociada con este fármaco, en el control de la HV activa.

\section{PACIENTES y MÉTOdOS}

Se incluyeron en forma prospectiva todos los pacientes ingresados por HV desde octubre de 2000 hasta febrero de 2003 a las Unidades de Cuidados Intensivos de la Clínica Las Condes y de los Hospitales Clínico de la Universidad de Chile y Militar de Santiago.

Criterios de inclusión: todo paciente cirrótico con: 1) episodio de HV activa. Se definió como sangrado activo a todo episodio que presente a lo menos uno de los siguientes criterios: a) hematemesis o melena en las $3 \mathrm{~h}$ previas; b) hematemesis 0 melena $>3 \mathrm{~h}$, pero con una presión arterial sistólica $<80 \mathrm{mmHg}$; y c) presencia en la endoscopia de sangre fresca en el estómago, sin otra causa que explique el sangrado, más que los várices sangrantes; y 2) panendoscopia precoz (dentro de las 12 h). El sitio de sangrado por várices esofágicas fue definido según la presencia de alguno de los siguientes criterios: 1) sangrado por várices activo, en chorro; 2) sangrado activo en napa; 3 ) signos de sangrado reciente (coágulo adherido plano o elevado); y 4) várice sin punto de rotura evidente, pero con sangre fresca o digerida en estómago, en ausencia de otras lesiones ${ }^{22}$.

Criterios de exclusión: aquellos pacientes que presentaran al menos uno de los siguientes criterios: 1) edad mayor o igual a 80 años; 2) hipertensión portal no cirrótica; 3) hepatocarcinoma; 4) escleroterapia reciente (dentro de los últimos 15 días); 5) taponamiento con balón o uso de drogas vasoactivas (dentro de los 8 últimos días); 6) falla hepática terminal (síndrome hepatorrenal tipo 10 etapa terminal de la cirrosis hepática [CH]); 7) pacientes cirróticos que presentaron un episodio de hemorragia digestiva alta, pero sin los criterios de sangrado activo definidos anteriormente; y 8) pacientes cirróticos que pese a haber presentado un sangrado activo y haber recibido tratamiento inicial con octreótido, el estudio endoscópico confirma una etiología no variceal.

Se definió fracaso del tratamiento cuando existía la presencia de al menos uno de los criterios de la clasificación de Baveno III ${ }^{22}$ : 1) dentro de las primeras $6 \mathrm{~h}$ : a) transfusión $\geq 4$ unidades de glóbulos rojos e incapacidad de 
mantener la presión arterial media (PAM), >70 mmHg o aumentar la presión sistólica, $>20 \mathrm{mmHg}$ sobre su valor basal; y b) incapacidad de reducir la frecuencia cardíaca (FC), <100 latidos por min 0 disminuir su valor basal, <20 latidos; 2) después de las 6 h: a) hematemesis; b) disminución de la PAM >20 mmHg; y aumento de la FC >20 latidos por min en 2 controles separados por $1 \mathrm{~h}$; y c) transfusión $>2$ unidades de glóbulos rojos para mantener hematocrito de $27 \%$ o de hemoglobina en $9 \mathrm{~g} / \mathrm{dl}$.

Los pacientes fueron transfundidos solamente si la hemoglobina era menor a $8 \mathrm{~g} / \mathrm{dl}$. En caso de resangrado, éstos fueron reevaluados con endoscopia dentro de las $24 \mathrm{~h}$. El control de la hemorragia fue definido por los siguientes criterios ${ }^{17}: 1$ ) ausencia de un nuevo episodio de hematemesis; 2) presión arterial estable, sin caída de la presión arterial sistólica mayor a $20 \mathrm{mmHg}$, una vez que la presión arterial se había estabilizado; 3) FC estable, sin aumento de la frecuencia mayor a 20 por min, una vez estabilizada; y 4) requerimientos transfusionales no mayores a 2 unidades de glóbulos rojos para mantener hematocrito y hemoglobina mayor a $27 \%$ y $9 \mathrm{~g} / \mathrm{dl}$, respectivamente.

$\mathrm{Al}$ ingreso al hospital (tiempo 0), se consignaron los siguientes elementos: antecedentes clínicos, anamnesis, examen físico, hemograma, velocidad de sedimentación, pruebas hepáticas, protrombina, función renal, electrolitos plasmáticos, glicemia y orina completa. La severidad de la $\mathrm{CH}$ fue definida según la clasificación de Child-
Pugh $^{23}$. Todos los pacientes recibieron profilaxis antibiótica (ciprofloxacino o ceftriaxona por 7 días). En caso de existir ascitis, se efectuó, previo al inicio de los antibióticos, una punción del líquido para descartar peritonitis bacteriana espontánea.

Protocolo. Una vez ingresados a la Unidad de Pacientes Críticos, los pacientes fueron asignados al azar y de acuerdo a la disponibilidad del centro asistencial en tres grupos. El grupo 1 recibió, previo a la endoscopia de urgencia, octreótido en bolus $50 \mu \mathrm{g}$ en $10 \mathrm{ml}$ de solución salina isotónica intravenosa, seguido de una infusión continua de $50 \mu \mathrm{g} / \mathrm{h}$ (12 ampollas en $250 \mathrm{ml}$ de solución salina isotónica intravenosa a $10 \mathrm{ml} / \mathrm{h}$ ) por 5 días, si el paciente cumplía los criterios de inclusión. La endoscopia de urgencia se realizó dentro de las primeras $12 \mathrm{~h}$ del ingreso al hospital (tiempo 0), efectuándose escleroterapia (monoetanolamina al $5 \%$, dosis total de $5 \mathrm{ml}$ por sesión) o ligaduras según la experiencia del operador. El grupo 2 recibió sólo tratamiento con octreótido, en las mismas condiciones que el grupo mixto. El grupo 3 se trató sólo endoscópicamente (esclerosis 0 ligaduras).

En caso de persistencia o recurrencia del sangrado, los pacientes fueron sometidos a esclerosis o ligadura endoscópica, y de persistir éste se les colocaba TIPS (pacientes Child-Pugh B y C) 0 cirugía derivativa (Child-Pugh A) (Figura 1). Los pacientes fueron evaluados por complicaciones,

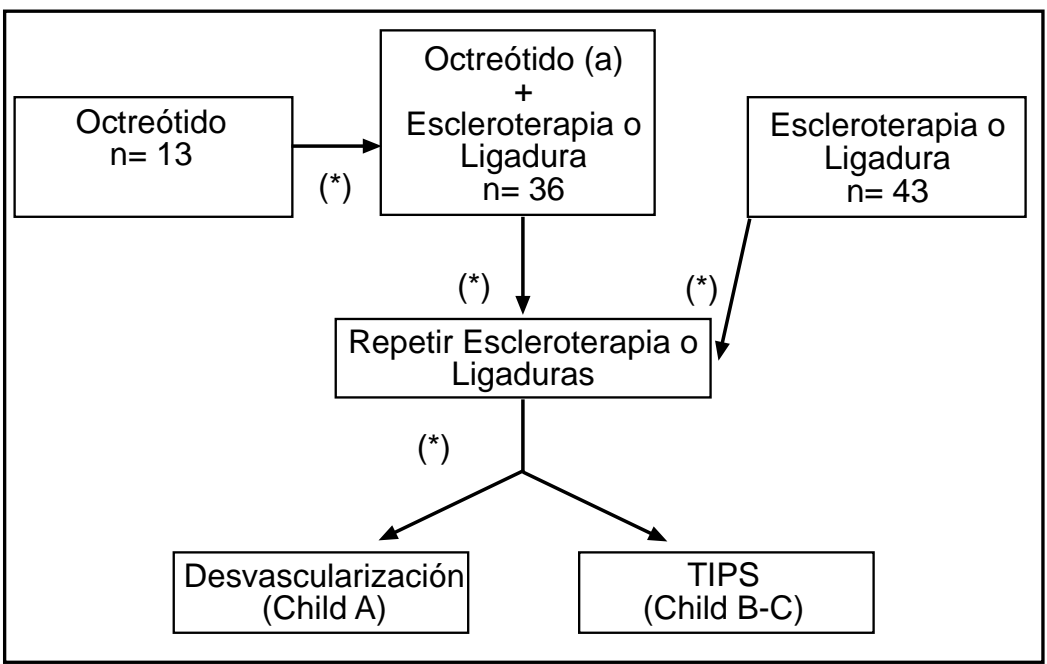

Figura 1. Protocolo seguido en el tratamiento de los pacientes cirróticos con hemorragia variceal activa (a) Octreótido $50 \mu \mathrm{g}$ (bolus) $+50 \mu \mathrm{g} / \mathrm{h}$ (infusión continua por 5 días).

(*) Fracaso a tratamiento. 
requerimientos transfusionales, días de hospitalización y mortalidad hasta los 42 días de ingresados.

Análisis estadístico. A las variables estudiadas se les calculó promedio \pm desviación estándar en el caso de variables continuas y porcentaje en el caso de variables categóricas. El análisis estadístico se realizó mediante ANOVA de una vía cuando se trataba de variables continuas y chi cuadrado y test de Kruskal-Wallis, en caso de variables nominales o categóricas. En todos los casos se usó un nivel de significación estadística de $\mathrm{p}<0,05$. También se practicó un análisis de sobrevida, donde los grupos fueron evaluados mediante el test Log-rank, con nivel de significación estadística de $p<0,05$. Se usó el test de Log-rank debido a que existían más de dos grupos. Los datos fueron graficados mediante la curva de sobrevida de Kaplan-Meier.

\section{RESULTADOS}

Se incluyeron 92 pacientes con $\mathrm{HV}$ activa, 56 provenientes del Hospital Clínico de la Universidad de Chile, 18 de la Clínica Las Condes y 18 del Hospital Militar. Cincuenta y tres eran hombres

Tabla 1. Características de 92 pacientes con hemorragia variceal activa tratados con octreotido o tratamiento endoscópico o ambos (escleroterapia o ligadura)

\begin{tabular}{|c|c|c|c|c|}
\hline & $\begin{array}{c}\text { Grupo } 1 \\
\text { Octreótido }+ \\
\text { tratamiento } \\
\text { endoscópico* } \\
n=36\end{array}$ & $\begin{array}{c}\text { Grupo } 2 \\
\text { Octreótido } \\
n=13\end{array}$ & $\begin{array}{c}\text { Grupo } 3 \\
\text { Tratamiento } \\
\text { endoscópico* } \\
\text { n=43 }\end{array}$ & $\mathrm{p}$ \\
\hline $\begin{array}{l}\text { Edad (años; promedio } \\
\quad \pm \text { desviación estándar) }\end{array}$ & $60,2 \pm 10,5$ & $63,4 \pm 13,1$ & $61,2 \pm 9,4$ & $(0,65)$ \\
\hline $\operatorname{Sexo}(\mathrm{M} / \mathrm{F})$ & $20 / 16$ & $4 / 9$ & $30 / 13$ & 0,039 \\
\hline Etiología: & & & & $(0,085)$ \\
\hline Alcohol & 14 & 6 & 32 & \\
\hline Criptogénica & 14 & 6 & 5 & \\
\hline $\mathrm{VHC}$ & 2 & - & 4 & \\
\hline EHNA & 3 & - & 1 & \\
\hline Hepatitis autoinmune & 2 & 1 & 1 & \\
\hline Hemocromatosis & 1 & - & - & \\
\hline \multicolumn{5}{|l|}{ Child-Pugh: } \\
\hline A & 6 & 3 & 15 & \\
\hline B & 22 & 7 & 12 & \\
\hline $\mathrm{C}$ & 8 & 3 & 16 & \\
\hline $\mathrm{X} \pm \mathrm{DS}$ & $8,2 \pm 1,9$ & $8,8 \pm 2,7$ & $9,0 \pm 2,5$ & $(0,12)$ \\
\hline $\begin{array}{l}\text { Características endoscópicas } \\
\text { del sitio del sangrado: }\end{array}$ & & & & $(0,2)$ \\
\hline 1 & 6 & 0 & 2 & \\
\hline 2 & 9 & 2 & 11 & \\
\hline 3 & 16 & 8 & 27 & \\
\hline 4 & 5 & 3 & 3 & \\
\hline
\end{tabular}

VHC: virus hepatitis C; EHNA:esteatohepatitis no alcohólica. Características endoscópicas del sitio de sangrado: 1 activo en chorro, 2 activo en napa, 3 coágulo adherido plano o elevado, y 4 sangre intraluminal, sin punto de ruptura evidente de la várice.

*Tratamiento endoscópico = esclerosis o ligadura. 
(57,6\%) (edad promedio \pm DS: $61 \pm 10$ años) y 39 mujeres (42,4\%) (edad promedio \pm DS: $61 \pm 11$ años). La etiología fue alcohólica en 52 pacientes (56,5\%), criptogénica en 25 (27\%), virus $C$ en 6 $(6,5 \%)$, esteatohepatitis en $4(4,3 \%)$, hepatitis autoinmune en $4(4,3 \%)$, y hemocromatosis en un paciente. No hubo diferencias significativas en la edad y en el puntaje de Child-Pugh, aunque sí hubo diferencias significativas al comparar el sexo entre los tres grupos $(\mathrm{p}<0,039)$ (Tabla 1).

Las características endoscópicas del sitio de sangrado por várices esofágicas se resumen en la Tabla 1, sin que se observen diferencias en sus hallazgos al ingreso, siendo la presencia de coágulo adherido el más frecuente en los tres grupos. El sangrado por várices fue controlado en 77 pacientes (84\%). De los 15 pacientes que resangraron, 3 sangraban en chorro, 5 en napa y 7 con coágulo adherido. En ningún caso hubo dudas del origen del sangrado. El número total de pacientes que resangró a las $24 \mathrm{~h}$ y a los 5 días según la modalidad de tratamiento, se detalla en la Tabla 2. De los 15 pacientes que resangraron, 3 eran del grupo mixto, 4 del grupo octreótido y 8 del grupo endoscópico. Todos ellos fueron tratados según protocolo (Figura 1). Tres pacientes requirieron finalmente la colocación de un TIPS, dos del grupo mixto y uno del grupo octreótido. En ningún paciente se realizó cirugía derivativa.

El promedio de unidades de glóbulos rojos transfundidas fue similar en cada grupo: Grupo 1: de 0 a 9 unidades; Grupo 2: De 2 a 6 unidades y Grupo 3: de 0 a 11 unidades (Tabla 2).

Los tipos de complicaciones observadas, se resumen en la Tabla 2. En el grupo con terapia mixta la complicación más frecuente fue infecciosa (10 casos), y de ellos 8 correspondieron a neumonía. En el grupo tratado sólo con octreótido, presentaron neumonía 2 pacientes. Finalmente, en el grupo que recibió terapia endoscópica, las infecciones se presentaron en 9 casos de los cuales 8 correspondieron a peritonitis bacteriana (Tabla 2).

Los días de hospitalización fueron similares en los tres grupos y sin diferencias estadísticas (Tabla 2). Fallecieron 11 pacientes, sin alcanzar significación estadística entre los grupos según la curva de

Tabla 2. Resultados en pacientes cirróticos con hemorragia variceal activa. Según tipo de tratamiento

\begin{tabular}{|c|c|c|c|c|c|c|c|}
\hline & \multicolumn{2}{|c|}{$\begin{array}{c}\text { Grupo } 1 \\
\text { Octreótido }+ \\
\text { tratamiento } \\
\text { endoscópico* } \\
n=36\end{array}$} & \multicolumn{2}{|c|}{$\begin{array}{c}\text { Grupo } 2 \\
\text { Octreótido } \\
n=13\end{array}$} & \multicolumn{2}{|c|}{$\begin{array}{c}\text { Grupo } 3 \\
\text { Tratamiento } \\
\text { endoscópico* } \\
\text { n=43 }\end{array}$} & \multirow[t]{2}{*}{$\mathrm{P}$} \\
\hline & $\mathrm{n}$ & $\%$ & $\mathrm{n}$ & $\%$ & $\mathrm{n}$ & $\%$ & \\
\hline \multicolumn{8}{|l|}{ Hemostasia: } \\
\hline A las $24 \mathrm{~h}$ & 35 & $(97,2)$ & 9 & $(69,2)$ & 40 & $(93,0)$ & $(0,2)$ \\
\hline A los 5 días & & $(91,7)$ & 9 & $(69,2)$ & 35 & $(81,4)$ & $(0,15)$ \\
\hline $\begin{array}{l}\text { Transfusión de GR } \\
\text { (unidades) }\end{array}$ & \multicolumn{2}{|c|}{$3,2 \pm 2,3$} & \multicolumn{2}{|c|}{$3,4 \pm 1,4$} & \multicolumn{2}{|c|}{$2,8 \pm 2,5$} & $(0,6)$ \\
\hline \multicolumn{5}{|l|}{ Complicaciones: } & \multicolumn{3}{|c|}{$(0,45)$} \\
\hline $\mathrm{N}^{\circ}$ total de pacientes & 15 & $(41,7)$ & 4 & $(30,8)$ & 17 & $(39,5)$ & \\
\hline Insuficiencia hepática & 8 & $(29,6)$ & 3 & (60) & 10 & $(52,6)$ & \\
\hline Infección & 10 & (37) & 2 & $(40)$ & 9 & $(47,4)$ & \\
\hline Insuficiencia renal & 9 & (33) & - & & - & & \\
\hline Días de hospitalización & \multicolumn{2}{|c|}{$10,9 \pm 6,2$} & \multicolumn{2}{|c|}{$11,8 \pm 7,4$} & \multicolumn{2}{|c|}{$\begin{array}{l}10,9 \pm 5,8 \\
8\end{array}$} & $(0,89)$ \\
\hline Mortalidad a los 42 días & 1 & $(2,8)$ & 2 & $(15,4)$ & 8 & $(18,6)$ & $(0,089)$ \\
\hline
\end{tabular}

*Tratamiento endoscópico=esclerosis o ligadura. 
Kaplan-Meier $(0,089)$ (Figura 2). Uno en el grupo mixto (2,8\%), (Child-Pugh C); 2 en el grupo octreótido (15,4\%) (un caso Child-Pugh B y el otro C); y 8 en el grupo endoscópico (18,6\%), todos Child-Pugh C (Tabla 2). El 93\% de las muertes ocurrieron en los primeros 5 días de iniciada alguna modalidad terapéutica.

La curva de Kaplan-Meier muestra que el porcentaje de pacientes sin hemorragia a los 42 días fue de 91,7\% en el grupo mixto; $69,2 \%$ en el grupo octreótido; y 79,1\% en el grupo endoscópico, sin alcanzar significación estadística $(\mathrm{p}=0,14)$ (Figura 3). Ninguno de los pacientes del grupo mixto o del grupo con octreótido presentó efectos adversos atribuibles al fármaco.
No se encontraron diferencias significativas entre los tres centros participantes con respecto al control de la hemorragia o la mortalidad en los pacientes tratados.

\section{DISCUSIÓN}

La HV es una complicación seria en pacientes con $\mathrm{CH}$ y está asociada con una elevada mortalidad, la que aumenta en 30\% cuando hay recurrencia ${ }^{1-4}$. Por lo tanto, cualquier tratamiento que pretenda controlarla deberá ser evaluado considerando la prevención del resangrado ${ }^{1,2}$. La eficacia de la escleroterapia y de la ligadura endoscópica en

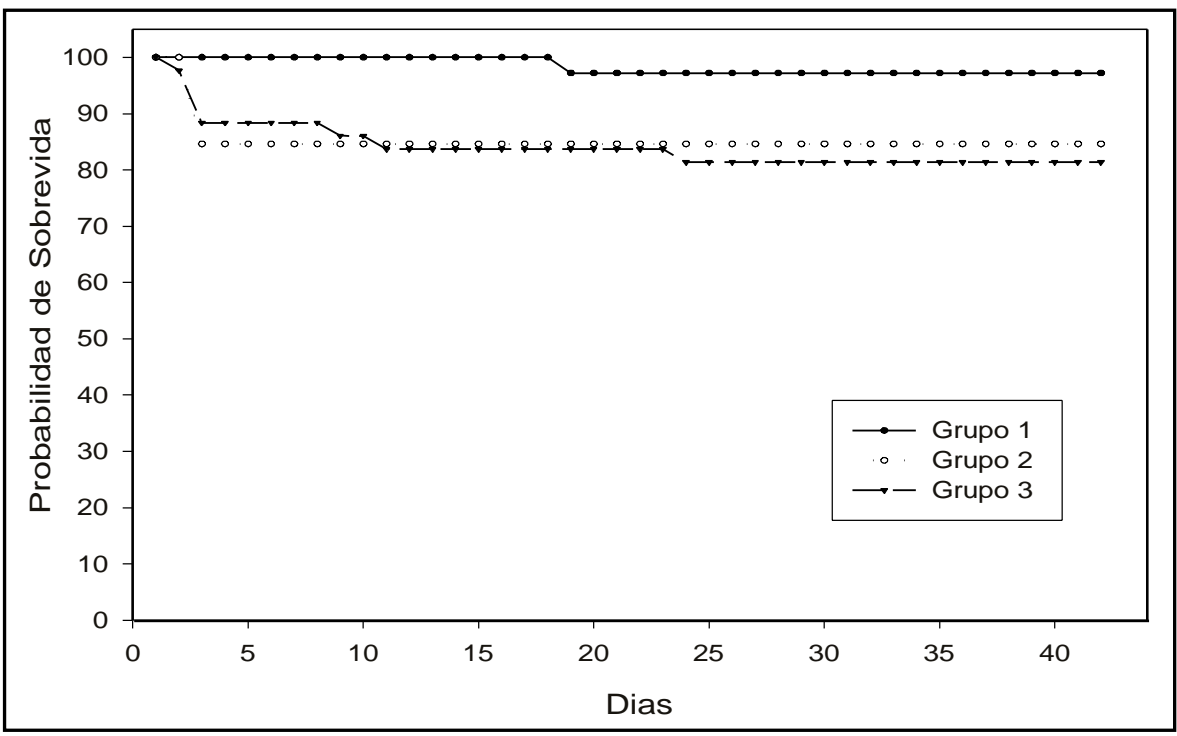

FiguRa 2. Estimación de la sobrevida de los pacientes a los 42 días para las tres modalidades de tratamiento según curva de Kaplan-Meier.

Grupo 1: tratamiento mixto (endoscópico y octreótido); Grupo 2: tratamiento con octreótido; y Grupo 3: tratamiento endoscópico. $(p=0,089$ entre los tres grupos).

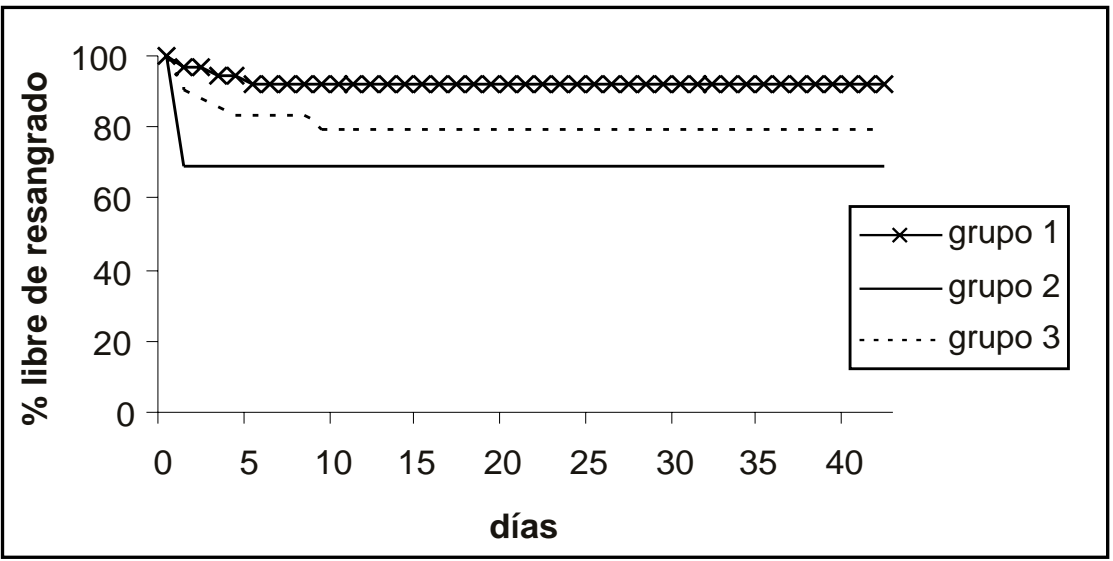

Figura 3. Proporción acumulada de pacientes libres de resangrado para las tres modalidades de tratamiento a los 42 días según curva de KaplanMeier.

Grupo 1: tratamiento mixto (endoscópico y octreótido); Grupo 2: tratamiento con octreótido; y Grupo 3: tratamiento endoscópico. $(p=0,14$ entre los tres grupos). 
controlar la HV aguda con baja morbilidad y mortalidad, permitirían sugerir que las técnicas endoscópicas podrían ser el tratamiento con el cual puedan compararse otras modalidades terapéuticas $^{2,24}$. Por lo tanto, el presente estudio fue diseñado para comparar la eficacia de la administración de octreótido sólo o asociado con tratamiento endoscópico contra la esclerosis o ligadura variceal en el control de la HV activa y en la prevención del resangrado precoz. Si bien no se observaron diferencias significativas en la hemostasia inicial ( $24 \mathrm{~h})$, y a los 5 días, como en la necesidad de transfusión, número de complicaciones secundarias a la $\mathrm{CH}$ y días de hospitalización, sí existiría una tendencia a una mayor probabilidad de sobrevida a los 42 días en el grupo tratado con terapia mixta comparado con los otros dos grupos $(p=0,089)$ (Tabla 2) (Figura 2).

En los últimos años ha existido gran interés en el uso de drogas vasoactivas en el tratamiento de la HV. Diferentes estudios controlados y randomizados han sugerido que el octreótido, un análogo de la somatostatina con diferente afinidad a sus receptores, podría ser una terapia adyuvante al tratamiento endoscópico en la HV ${ }^{14,15,25}$. Pese a los resultados de estos estudios, el mecanismo de acción del octreótido permanece aún desconocido. Recientemente Escorsell y col ${ }^{16}$ han señalado que el efecto del octreótido sobre la presión portal y el flujo de la vena ácigos sólo sería transitorio y no se correlacionaría con el efecto prolongado que posee el octreótido sobre los niveles plasmáticos de glucagón. Sanyal ${ }^{26}$ ha sugerido, que el beneficio del octreótido estaría dado por un efecto sobre los sistemas neurohumorales que regulan el flujo sanguíneo mesentérico, como el aumento del flujo esplácnico y de la presión portal dado por el contenido de sangre intragástrico ${ }^{19,20,27}$. Estos hallazgos podrían explicar, al menos en parte, las discrepancias en los resultados obtenidos en los distintos estudios comparándolo con otras drogas como vasopresina y terlipresina $21,28,29$.

Sin duda, el uso clínico del octreótido sigue siendo hasta ahora objeto de controversia. En parte, por su elevado costo en comparación con las técnicas endoscópicas y porque los estudios realizados hasta ahora difieren considerablemente en su metodología, como es el caso de la severidad de los pacientes con $\mathrm{CH}$ (porcentaje de pacientes más deteriorados), dosis, duración y vía de administración del fármaco ${ }^{15,30-37}$. Pese a esto, recientemente en tres metaanálisis se ha sugerido que aunque el tratamiento con octreótido no modifica la mortalidad por $\mathrm{HV}$, sí mejoraría la eficacia del tratamiento endoscópico para lograr el control de la hemorragia y la hemostasia al quinto día, e incluso sería tan seguro y efectivo como vasopresina y terlipresina ${ }^{21,28,29}$. En el presente estudio, sólo existe una tendencia a una menor mortalidad a los 42 días en el grupo tratado con terapia mixta (Tabla 2) (Figura 2). Si este resultado se mantuviera con un número de 100 pacientes en cada grupo, las diferencias habrían resultado significativas. Nuestros resultados difieren con los de otras publicaciones. Es así como Zuberi y colli5, quien usando las mismas dosis y duración de octreótido, señalan que la combinación de escleroterapia y octreótido disminuiría significativamente la frecuencia de resangrado, transfusiones y estadía hospitalaria. Sin embargo, este estudio tiene diferencias en la metodología que pueden explicar dichos resultados, como excluir pacientes cirróticos Child $\mathrm{C}$, incluir pacientes con várices gástricas y gastropatía hipertensiva portal y tener un tiempo de observación de sólo 5 días. Sung y cols $^{31}$ también han señalado que la terapia mixta disminuiría la recurrencia de sangrado. En su estudio cerca de $40 \%$ de los pacientes eran ChildC. Esta cantidad es superior al $29 \%$ de pacientes Child-C ingresados en nuestro estudio (Tabla 1). Por otro lado, se hace difícil comparar nuestros resultados con estudios como el de Freitas y col ${ }^{36}$, quienes señalan que el uso de octreótido $25 \mu \mathrm{g} / \mathrm{hr}$ iv por $48 \mathrm{~h}$ asociado a escleroterapia mejoraría la hemostasia inicial, hemostasia a las $48 \mathrm{~h}$ y requerimientos transfusionales. Esto mismo sucede con el estudio de D'Amico y $\mathrm{Col}^{14}$, quienes señalan que el uso de octreótido $100 \mu \mathrm{g}$ subcutáneo 3 veces/ día por 15 días, asociado a betabloqueadores 0 escleroterapia disminuiría el riesgo de resangrado.

En nuestro estudio, la diferencia en el número de pacientes ingresados en cada uno de los tres grupos, particularmente en el grupo tratado sólo con octreótido (13 pacientes), se debió a una baja tendencia inicial de hemostasia a las $24 \mathrm{~h}$ y a los 5 días en comparación con el grupo tratado con terapia mixta y el grupo tratado solo endoscópicamente y comparables a los observados espontáneamente, sin mediar terapia específica, lo que 
hizo necesario suspender la inclusión de nuevos casos en este grupo ${ }^{1-4}$. Aunque finalmente no hubo una diferencia estadísticamente significativa (Tabla 2), probablemente por el número de pacientes incluidos, nosotros podemos sugerir que el tratamiento con octreótido no debe reemplazar el tratamiento endoscópico en estos casos.

Aunque se ha señalado que cualquier muerte que ocurra antes del día 42 debería relacionarse con el episodio de sangrado ${ }^{2}$, el hecho que en nuestro estudio el 93\% de las muertes y el $94 \%$ de los resangrados hayan ocurrido dentro de los primeros 5 días de iniciada cualquier modalidad terapéutica, nos permite confirmar que cualquier terapia que se quiera evaluar en el tratamiento de la HV, deberá realizarse principalmente en este período de tiempo. Esto explica porqué algunos estudios han sugerido que el tratamiento con octreótido y otras drogas vasoactivas deberían tener una duración de 5 días ${ }^{15,30,31}$.

La evaluación de una nueva terapia no sólo requiere demostrar su eficacia, sino también sus potenciales efectos adversos. En nuestro estudio, no hubo efectos adversos secundarios al uso de octreótido, por lo que la terapia con este fármaco nos parece segura.

Hasta la fecha, a nivel nacional existen muy pocos estudios que hayan evaluado el tratamiento

\section{REFERENCIAS}

1. Chang L, Sung J. Review article: The role of pharmaco-therapy for acute variceal haemorrhage in the era of endoscopic haemostasis. Aliment Pharmacol Ther 1997; 11: 45-50.

2. Grace ND, Groszmann RJ, Garcia-Tsao G, Burrougs aK, Paglaro L, Makuch RW et al. Portal hypertension and variceal bleeding: an AASLD single topic symposium. Hepatology 1998; 28: 868-80.

3. LuKetic V, SANYAL A. Esophageal varices. Clinical presentation, medical therapy and endoscopy therapy. Gastroenterol Clinics of North America 2000; 29: 337-85.

4. Sharara A, Rockey D. Gastroesophageal variceal hemorrhage. N Engl J Med 2001; 345: 669-81.

5. Helmy A, Hayes P. Review article: current endoscopic therapeutic options in the management of endoscópico de la HV activa y ningún trabajo que haya evaluado el uso de algún fármaco asociado al tratamiento endoscópico como es el caso del octreótido. En este estudio, en el grupo tratado sólo con endoscopia, logramos una hemostasia a las $24 \mathrm{hr}$ y a los 5 días en 93 y 81,4\% de los pacientes respectivamente, porcentajes que se encuentra dentro de 80 a $90 \%$ de éxito descrito en estudios internacionales ${ }^{12,13}$. Sin embargo, estos resultados son algo inferiores al 96\% señalado por Vargas y $\mathrm{col}^{11}$, probablemente porque los pacientes sometidos a terapia de urgencia no son comparables, al incluir este último autor en el grupo que recibió tratamiento endoscópico de urgencia, pacientes que habían sangrado en un plazo de hasta tres días antes de su ingreso, desconociéndose además antecedentes sobre transfusiones y días de hospitalización.

En conclusión, aunque nuestros resultados no fueron estadísticamente significativos, la tendencia observada permitiría recomendar el uso de octreótido en infusión intravenosa como coadyuvante al tratamiento endoscópico en el manejo de la HV. Sin embargo, un mayor número de pacientes podría mejorar aspectos estadísticos que no alcanzaron significación en nuestro estudio.

variceal bleeding. Aliment Pharmacol Ther 2001; 15: 574-94.

6. Silva G, Fluxá F, Brahm J, Segovia R, Quesada S, Silva $C$ et al. Aspectos evolutivos de la hipertensión portal en pacientes cirróticos. Gastr Latinoam 2002, 13; 4: 263-9.

7. Jalan R, Hayes P. UK guidelines on the management of variceal haemorrhage in cirrhotic patients. Gut 2000; 46 (Suppl III): iii 1-15.

8. BuRroghs A. Pharmacological treatment of acute variceal bleeding. Digestion 1998; 59: 28-36.

9. Burroghs A, Planas R, Svodova P. Optimizing emergency care of upper gastrointestinal bleeding in cirrhotic patients. Scand J Gastroenterol 1998; 33 Supl 226: 14-24.

10. LAINE L, Cook D. Endoscopic ligation compared with sclerotherapy for treatment of esophageal variceal bleeding. Ann Intern Med 1995; 123: 280-7. 
11. Vargas L, Morales X, Ovalue L, Estay R, Soto JR. Ligadura elástica complementada de esclerosis en el tratamiento de várices esofágicas. Experiencia en la hemorragia activa y en el tratamiento electivo. Rev Méd Chile 2001; 129: 1387-94.

12. Hartigan PM, Gebhard RL, Gregory PB For the VACVSG. Sclerotherapy for actively bleeding esophageal varices in male alcoholics with cirrhosis. Gastrointest Endosc 1997; 46: 1-7.

13. Laine L, El-Newihi HM, Migikovsky B, Sloane R, García F. Endoscopic ligation compared with sclerotherapy for treatment of bleeding esophageal varices. Ann Intern Med 1993; 119: 1-7.

14. D’Amico G, Poloti F, Morabito A, D’Antoni A, GuerRera D ET aL. Octreotide compared with placebo in a treatment strategy for early rebleeding in cirrhosis. A double blind, randomized pragmatic trial. Hepatology 1998; 28: 1206-14.

15. ZuBerI B, BaLOCH Q. Comparison of endoscopic variceal sclerotherapy alone and in combination with octreotide in controlling acute variceal hemorrhage and early rebleeding in patients with low-risk cirrhosis. Am J Gastroenterol 2000, 95: 768-71.

16. Escorsell A, Bandi JC, Andreu V, Moitinho E, Garcia-Pagan JC, Bosch J et al. Desensitization to the effects of intravenous octreotide in cirrhotic patients with portal hypertension. Gastroenterology 2001; 120: 161-9.

17. Zhang HB, Wong B, Zhou XM, Guo XG, Zhao SJ, WANG JH. Effects of somatostatin, octreotide and pitressin plus nitroglicerine on systemic and portal haemodynamics in the control of acute variceal bleeding. Int J Clin Pract 2002; 56: 44751.

18. SADOwSKI D. Use of octreotide in the acute management of bleeding esophageal varices. Can J Gastroenterol 1997; 11: 339-43.

19. Sabba C, Buonamico P, Vendemiale G, Berardi E, Antonica G, Palmieri V et al. Haemodynamic effects of propanolol, octreotide and their combination during fasting and post-prandial splanchnic hyperaemia in patients with cirrhosis. Eur J Gastroenterol Hepatol 2001; 13: 163-9.

20. Ludwig D, Schadel S, Buruning A, Schiefer B, Stange EF. 48-hour haemodinamyc effects of octreotide on postprandial splanchnic hyperaemia in patients with liver cirrhosis and portal hypertension: doubled-blind, placebo-controlled study. Dig Dis Sci 2000; 45: 1019-27.

21. Corley D, Ce山o J, Adkisson W, Ko WF, KerLikoeske. Octreotide for acute esophageal variceal bleeding: A meta-analysis. Gastroenterology 2001; 120: 946-54.

22. FRANCHIS R. Updating concensus in portal hypertension: Report of the Baveno III concensus workshop on definitions, methodology and therapeutic strategies in portal hypertension. J Hepatol 2000; 33: 846-52.

23. Pugh RN, Murray-Lyon IM, Dawson JL, Pietroni MC, Wiwams R. Transection of the oesophagus for bleeding oesophageal varices. Br J Surg 1973; 60: 646-9.

24. Bohnacker S, Maydeo A, Soehendra N. Variceal bleeding and portal hypertension: Still a therapeutic challenge? Endoscopy 1999; 3: 26-37.

25. Besson I, Ingrand P, Person B, Boutrox D, HeresBASH D, BERNARD P. Sclerotherapy with or without octreotide for acute variceal bleeding. New Engl J Med 1995; 333: 555-60.

26. Sanyal A. Octreotide and its effects on portal circulation. Gastroenterology 2001; 120: 303-5.

27. Chen L, Groszmann RJ. Blood in the gastric lumen increases splanchnic blood flow and portal pressure in portal-hypertensive rats. Gastroenterology 1996; 111: 1103-10.

28. ERSTAD B. Octreotide for acute variceal bleeding. Ann Pharmacother 2001; 35: 618-26.

29. Bañares R, Albilos A, Rincon D, Alonso S, GonzaIEZ M, RuIz L ET AL. Endoscopic treatment versus endoscopic plus pharmacologic treatment for acute variceal bleeding: a meta-analysis. Hepatology 2002; 35: 609-15.

30. Bildozola M, Kravetz D, Argonz J, Romero G, Sú́REZ A, JMELNITZKY A ET AL. Efficacy of octreotide and sclerotherapy in the treatment of acute variceal bleeding in cirrotic patients. A prospective, multicentric and randomized clinical trial. Scand J Gastroentreol 2000; 35: 419-24.

31. Sung JJ, Chung SC, Yung MY, Lai CW, LaY JY, Lee YT ET AL. Prospective randomized study of effect of octreotide on rebleeding from oesophageal varices after endoscopic ligation. Lancet 1995; 346: 1666-9.

32. Sung JJ, Chung SC, Lai CW, Chan FLK, Leung JWC, YUNG MY ET AL. Octreotide infusion or emergency sclerotherapy for variceal haemorrhage. Lancet 1993; 18: 61-5. 
33. BuRRoghs A. Double blind randomized controlled trials of 5 day octreotide versus placebo associated with ES for trial failures. Hepatology 1996; 24: 252 (Abstract).

34. Jenkins S, Shields R, Davies M, Euias E, Turnbull A, BASSENDINE M ET AL. A multicentre randomized trial comparing octreotide and injection sclerotherapy in the management and outcome of acute variceal haemorrhage. Gut 1997; 41: 526-33.

35. Sivri B, Oksuzuglu G, Bayraktar Y, Kaythan B. A prospective randomized trial from Turkey com- paring octreotide versus injection sclerotherapy in acute variceal bleeding. Hepato-gastroenterology 2000; 47: 168-73.

36. Freitas D, Sofia C, Pontes JM, Gregorio C, Cabral JP, ANDRADE P. Octreotide in acute bleeding esophageal varices: A prospective randomized study. Hepato-gastroenterology 2000; 47: 1310-14.

37. Cales P, Maslah C, Bernard B, Garnier PP, Silvain C, Szostak-Talbodec N et al. Early administration of vapreotide for variceal bleeding in patients with cirrhosis. N Engl J Med 2001; 334; 23-8. 